\title{
EMBARAZO ADOLESCENTE: UN PROBLEMA PAÍS
}

El 26 de septiembre recién pasado se conmemoró el Día Mundial de la Prevención del Embarazo Adolescente, efectuándose una jornada de reflexión sobre el tema, con representantes del Ministerio de Salud, CEMERA (Centro de Medicina Reproductiva y Desarrollo Integral del Adolescente), FIGIJ (Federación Internacional de Ginecología Pediátrica y de la Adolescencia), CELSAM (Centro Latinoamericano Salud y Mujer), entre otros.

Los cambios culturales hacen que la vida sexual se inicie cada vez más precozmente, lo que conlleva un aumento del embarazo adolescente, realidad que se presenta en países desarrollados y en desarrollo (1), con importantes consecuencias sociales como es la perpetuación de la pobreza, la deserción escolar, la estigmatización social, etc., y consecuencias médicas como es la mayor morbimortalidad materno-perinatal e infantil de las madres adolescentes y de sus hijos.

Chile, en 2005, reportó 230.831 nacidos vivos, de ellos, $935(0,41 \%)$ correspondieron a embarazos de adolescentes de 10-14 años y 35.143 (15,2\%) a adolescentes de 15-19 años, 5 de estas últimas fallecieron como consecuencia del proceso reproductivo (14,2/100.000 nv) (2). En la Tabla I, se presenta un resumen de la tendencia de la natalidad y de la tasa de fecundidad específica de las adolescentes menores y mayores, en el período 1990-2005; se aprecia que la tasa de fecundidad específica en adolescentes menores se mantiene estable y las mayores registran un importante descenso, estas cifras ubican al país en un lugar de privilegio dentro de las estadísticas latinoamericanas (3). Pese a lo anterior, el embarazo adolescente en Chile conlleva también un alto riesgo perinatal, especialmente por una mortalidad neonatal e infantil significativamente mayor $(4,5)$, asociadas especialmente a una mayor incidencia de recién nacidos menores de 2500 gramos e insuficiente red de apoyo social.

La mortalidad materna en países en desarrollo es un enorme problema de salud pública, no resuelto en la gran mayoría de ellos y el embarazo ado- lescente contribuye a ella significativamente. En Chile, la amplia cobertura de la atención profesional e institucional del embarazo y parto, hacen que nuestra realidad sea diferente a la mayoría de los países latinoamericanos. Es así que en un análisis reciente (5), la mortalidad materna en adolescentes menores, pese a su elevada tasa $(41,9 / 100.000$ nv) no fue significativa y en adolescentes mayores $(19,3 / 100.000 \mathrm{nv})$ fue significativamente menor al grupo control, explicado especialmente por una baja mortalidad por aborto (3,9/100.000 nv) (6). Estas cifras dan un diagnóstico de situación, para que las autoridades sanitarias del país tomen las medidas necesarias para que ninguna madre-niña o sus hijos mueran como consecuencia del proceso reproductivo o por falta de una red de apoyo social. Lo importante es ¿deseaban esas niñas embarazarse?, ¿hicimos algo efectivo como familia o país para evitarlo?

El embarazo adolescente en Chile se da con mayor frecuencia en estratos socio-económicos identificado con la pobreza (7) y asociado a una alta deserción escolar (8), todo esto perpetúa la pobreza y transforma el embarazo adolescente en un importante problema país.

Es urgente la necesidad de desarrollar estrategias que tengan credibilidad entre la/os adolescentes para lograr el objetivo básico que es la prevención del embarazo y sus consecuencias. Son múltiples los programas internacionales desarrollados con ese objetivo, sin embargo, una reciente revisión sistemática de estudios controlados y randomizados concluyó que las estrategias de prevención primaria no mostraron un retraso significativo del inicio de la actividad coital, no lograron mejorar el uso de anticonceptivos en adolescentes hombres y mujeres, no redujeron la tasa de fecundidad adolescente, y 4 de 5 programas de abstinencia mostraron un aumento de los embarazos en las parejas de participantes masculinos (9). Pese a lo cual, algunas experiencias nacionales, posteriores a ese estudio, han mostrado reducción de la tasa 
de embarazo adolescente $(10,11)$.

Los niños y niñas de Chile necesitan ser escuchados y requieren de nuestro apoyo. Debemos ayudarlos como familia y país, elaborando programas educacionales no solo para ellos, sino también para los padres, legisladores, equipos de salud y especialmente para los educadores, algunos de ellos ya en marcha $(12,13)$. Incorporemos objetivos biológicos y valóricos, de alta credibilidad, sin imposiciones, ni politización del tema. ¡Los niños de Chile nos lo demandan!

Dr Enrique Donoso S. Editor Jefe.

Revista Chilena de Obstetricia y Ginecología.

Tabla I

NATALIDAD Y TASA DE FECUNDIDAD ESPECÍFICA EN ADOLESCENTES DE 10-14 AÑOS Y 15-19 AÑOS, CHILE 1990-2005

\begin{tabular}{rrrrrrrr}
\hline \multicolumn{4}{c}{$10-14$ años } & \multicolumn{3}{c}{$15-19$ años } \\
año & PE & N V & TF & PE & NV & TF \\
\hline 1990 & 589.180 & 742 & 1,26 & 609.389 & 39.543 & 64,9 \\
1995 & 632.708 & 1.033 & 1,63 & 597.333 & 37.852 & 63,4 \\
2000 & 700.684 & 1.055 & 1,51 & 629.166 & 39.257 & 62,4 \\
2005 & 731.237 & 935 & 1,28 & 719.637 & 35.143 & 48,8 \\
\hline
\end{tabular}

PE: población estimada. NV: nacidos vivos. TF: tasa de fecundidad (x 1000 mujeres).

Fuente: Anuarios de Demografía y Estadísticas Vitales, INE 1990 - 2005.

\section{REFERENCIAS}

1. WHO. Adolescent pregnancy. Issues in adolescent health and development. 2004.

2. Instituto Nacional de Estadísticas. Estadísticas Vitales de Chile, 2005.

3. Flórez CE, Soto VE. Fecundidad Adolescente y Desigualdad en Colombia y la Región de América Latina y el Caribe. Reunión de expertos sobre población y pobreza en América Latina y el Caribe. 14 y 15 de Noviembre 2006, Santiago, Chile CEPAL, UNFPA. Hallado en: http://www.eclac.org/celade/noticias/paginas/5/27255/Florez_Soto.pdf

4. Donoso E, Becker J, Villarroel L. Embarazo en la adolescente menor de 15 años. Análisis de la última década del siglo XX. Rev Chil Obstet Ginecol 2001;66:3916.

5. Donoso E, Becker J, Villarroel L. Natalidad y riesgo reproductivo en adolescentes de Chile, 1990-1999. Rev Panam Salud Pública 2003;14(1):3-8.

6. Donoso E. Reducción de la mortalidad materna en Chile, de 1990 a 2000. Rev Panam Salud Pública 2004;15(5):326-30.

7. Molina R, Molina T, González T. Madres niñas-adolescentes de 14 años y menos. Un grave problema de salud pública no resuelto. Rev Méd Chile 2007;135:7986.

8. Molina M, Ferrada C, Pérez R, et al. Embarazo en la adolescencia y su relación con la deserción escolar. Rev Méd Chile 2004;132:65-70.

9. DiCenso A, Guyatt G, Willan A, Griffith L. Interventions to reduce unintended pregnancies among adolescents: systematic review of randomised controlled trials. BMJ 2002; 324(7351): 1426. Hallado en: http:// www.bmj.com/cgi/reprint/324/7351/1426.

10. Cabezón C, Vigil P, Rojas I, Leiva ME, Riquelme R, Aranda W, García C. Adolescent pregnancy prevention: An abstinence-centered randomized controlled intervention in a Chilean public high school. J Adolesc Health 2005;36(1):64-9.

11. Vigil $P$, Riquelme $R$, Rivadeneira $R$, Aranda W. Effects of TeenSTAR, an abstinence only sexual education program, on adolescent sexual behavior. Rev Med Chil 2005;133(10):1173-82.

12. Ministerio de Educación. Política de educación en sexualidad. Para el mejoramiento de la calidad de la educación. 4aㅡ edición. 2001. Hallado en: http://www.mineduc.cl/usuarios/edu.sexual/doc/200510041153520. POLITICA\%20EN\%20SEXUALIDAD\%20Y\%20AFEC TIVIDAD.pdf

13. Ministerio de Educación. Comisión de evaluación y recomendaciones sobre educación sexual (Serie Bicentenario). Informe no oficial. Marzo, 2005. Hallado en: http://www.mineduc.cl/biblio/documento/libro_sexualidad.pdf 